\title{
Normas de associación palabras como indicador de declive semántico en adultos mayores con y sin demencia
}

Alma Esperanza Ríos Ponce, Diana Iris Luna Umanzor, Natalia Arias-Trejo

\section{Resumen}

El envejecimiento forma parte del desarrollo de la vida humana y lleva consigo una serie de cambios físicos, cognitivos, biológicos y conductuales que se pueden considerar normales dentro de los parámetros de esta evolución. Cuando estos cambios producen dificultades cognitivas y afectan la independencia de la persona, existe la posibilidad de padecer una demencia. Alteraciones en áreas como la memoria y el lenguaje han sido estudiadas a través de distintos métodos como la tarea de asociación de palabras, que permite conocer la organización de la memoria semántica y ha sido usada para estudiar cambios en el lenguaje. La presente investigación tiene como objetivo la identificación de cambios en las relaciones léxicas en dos patologías, enfermedad de Alzheimer y demencia mixta, a través de este tipo de tarea. Los resultados indican mayor deterioro de las relaciones léxicas en los participantes con enfermedad de Alzheimer que en aquellos con demencia mixta respecto a los grupos controles. 
Palabras clave: Normas de asociación de palabras; demencia tipo Alzheimer; demencia mixta; envejecimiento típico.

\section{Introducción}

El envejecimiento humano se define como un proceso gradual y adaptativo de tipo biológico, psicológico y social que es resultado de cambios programados por la genética. A medida que esto sucede, ocurre una disminución en la eficiencia del funcionamiento de los órganos y sistemas del individuo. El cerebro no es la excepción y envejece al igual que el resto del organismo en el que se dan cambios anatómicos, fisiológicos y funcionales (Mendoza-Núñez, 2013).

Las alteraciones a nivel anatómico-cerebral que han sido bien estudiadas (Villa, Navarro y Villaseñor, 2017) son: 1) La reducción del volumen cerebral de algunas estructuras; 2) cambios en la sustancia blanca; 3) adelgazamiento de la corteza cerebral, y 4) reducción de la producción de dopamina.

En la demencia existe un deterioro persistente y global de las funciones intelectuales adquiridas previamente. Se origina por efecto de una lesión orgánica difusa o multifocal cerebral (Peña-Casanova, 2007). Puede alterar múltiples funciones corticales superiores que incluyen memoria, pensamiento, orientación, comprensión, cálculo, aprendizaje, lenguaje y juicio. Las alteraciones de la función cognitiva se acompañan a menudo, y en otras ocasiones vienen precedidas de un deterioro del control emocional o social de la conducta o de la motivación (Jurado et al., 2013). Dentro de las etiologías de la demencia se incluyen las degenerativas, vasculares, traumáticas, infecciosas, obstructivas, metabólicas, tóxicas y neoplásicas (Arango-Lasprilla, Fernández y Ardila, 2003).

La demencia por enfermedad de Alzheimer (EA) se caracteriza principalmente por un deterioro en la memoria tipo amnésico, es decir, dificultad para aprender y recordar nueva información, así como un trastorno progresivo del lenguaje, que inicia con anomia y que puede progresar a afasia fluente, alteraciones en las habilidades viso-espaciales y déficits en funciones ejecutivas (planeamiento, introspección y juicio) (Pradilla, 2004). 
A la combinación de Alzheimer con enfermedad vascular cerebral se ha denominado demencia mixta (componente cortical y subcortical) y representa $13-17 \%$ de los casos en el mundo. En general, tanto la enfermedad de Alzheimer (EA) como el deterioro cognitivo vascular están fuertemente relacionadas con la edad. Hoy día existe más evidencia de que la demencia mixta (MxD) ocupa la tercera parte del total de las causas de demencia (Cervantes, Alvarado, Navarro, Ávila, Gutiérrez, Arellano y Funes, 2017).

Como consecuencia de los déficits cognitivos que presentan las personas con enfermedad neurodegenerativa, la identificación de un perfil cognitivo distintivo es esencial para brindarles un tratamiento integral. Entre los dominios afectados por esta enfermedad se encuentra el lenguaje, el cual está íntimamente relacionado con la memoria a través de la memoria semántica. La memoria semántica hace referencia al conocimiento del mundo y del lenguaje. Es una memoria que organiza información relacionada con hechos, conceptos y lenguaje (Ballesteros, 1999). Sin embargo, su contenido es mucho más amplio (Tulving, 1983) porque también incluye asociaciones entre palabras, conceptos, símbolos y sus asociaciones, y hechos (Ballesteros, 1999).

Para conocer la forma en la que está organizada la memoria semántica se han realizado diversas tareas, entre ellas tareas de asociación léxica. Las Normas de Asociación de Palabras (NAP) son un corpus que describe la relación que existe entre dos palabras de acuerdo con personas que hablan una misma lengua. Para obtener estas relaciones léxicas se emplea la técnica de asociación libre, en la cual se presenta al sujeto una palabra estímulo y se le pide que produzca la primera palabra que venga a su mente cuando escuche la palabra estímulo, obteniendo una lista de palabras evocadas por el sujeto, así como el tiempo que tardó en emitir una respuesta (Clark, 1970). Posteriormente, se obtiene la fuerza de asociación (FA) entre dos palabras, input y output, dividiendo la respuesta, output, con frecuencia más alta entre el total de respuestas diferentes emitidas. Asimismo, se pueden obtener medidas como el total de respuestas diferentes proporcionadas (NA), las respuestas idiosincrásicas o únicas (RI), respuestas en blanco (RB), así como el grado de asociación que presentan dos palabras entre sí, la cual se puede caracterizar como alta, 
moderada, baja o nula (Callejas, Correa, Lupiáñez y Tudela, 2003; Salles et al., 2008). Por ende, el objetivo de esta investigación es identificar los cambios en las relaciones léxicas en dos patologías, enfermedad de Alzheimer y demencia mixt, a través de una tarea de asociación de palabras.

\section{Método}

El presente trabajo es un estudio de tipo descriptivo transversal, en el que se busca comparar las medidas previamente mencionadas en los grupos a evaluar.

\section{Participantes}

Para este estudio, se evaluó un total de siete participantes con demencia, los cuales fueron divididos en dos grupos: uno con cuatro participantes con demencia de tipo Alzheimer y otro con tres diagnosticados con demencia mixta. Cada uno de estos grupos fueron pareados con un grupo control que cumplía con la misma edad y escolaridad.

En el grupo experimental, los participantes seleccionados debían tener edades de 60 años en adelante al momento del estudio, cursar con diagnóstico de demencia por enfermedad de Alzheimer o demencia mixta clasificada con niveles leve y moderado, así como ser monolingües del español. Mientras que sus controles debían ser adultos mayores de 60 años en adelante sin diagnóstico de enfermedades neurodegenerativas y monolingües del español.

Se excluyeron aquellos pacientes que tuvieran historial de enfermedad neurológica u otro trastorno mental y de comportamiento, incluyendo aquellos participantes que presentasen diagnóstico de depresión o ansiedad sin tratamiento estable.

\section{Estímulos}

La selección de 234 palabras estímulo fue realizada con base en el trabajo de Barrón-Martínez y Arias-Trejo (2014), quienes crearon una base de datos de asociaciones léxicas en 
adultos jóvenes mexicanos. Estas palabras son sustantivos concretos seleccionados a partir de los MacArthur Inventarios del Desarrollo de Habilidades Comunicativas, Palabras y Oraciones (Jackson-Maldonado et al., 2003, citado en Barrón-Martínez y Arias-Trejo, 2014). Estas palabras son divididas al azar en dos listas, para evitar cansancio en el participante, y acomodadas de tal forma que dos palabras no pertenezcan a la misma categoría semántica para evitar interferencia entre palabras.

\section{Instrumentos}

Se utilizó un consentimiento informado sobre la confidencialidad y anonimidad de los datos proporcionados, así como un cuestionario sociodemográfico. Éstos podrían ser proporcionados por el cuidador primario, en el caso de los participantes con demencia.

Para la ejecución y recolección de la tarea de las normas de asociación se utilizó una laptop Dell Modelo Inspiron 14, 5000 series P49G y con ayuda de un programa llamado SS_Palabras 2.0 se presentaron 123 palabras estímulo.

\section{Procedimiento}

En el caso de los participantes con EA y con MxD se asistió a instituciones especializadas en el tratamiento de dicha enfermedad. Mientras que para el caso del grupo con envejecimiento típico se evaluó a adultos mayores sanos externos que consintieron la aplicación de la tarea.

Después de llenar el consentimiento informado y el cuestionario sociodemográfico, el participante realizó con el experimentador la tarea computarizada.

En primera instancia, se proporcionó a los participantes las instrucciones necesarias para poder ejecutar la tarea. Éstas consisten básicamente en nombrar una palabra (p. ej., gato) y pedirle que responda la primera palabra que venga a su mente.

El experimentador escribe la respuesta del participante en un cuadro correspondiente proporcionado por el programa (ver Figura 1). La tarea contiene tres ensayos de entrenamiento y 
120 ensayos de prueba. Por medio de una tecla, el reloj interno del sistema computacional registraba el tiempo de respuesta.

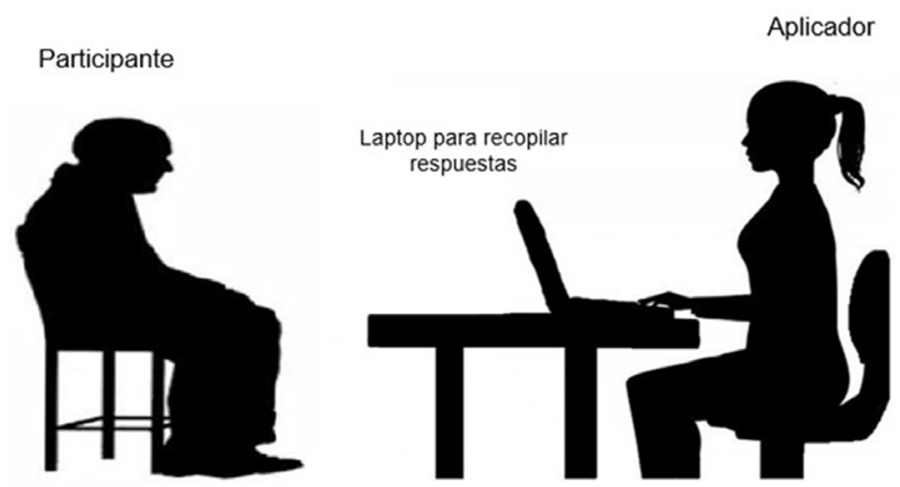

Figura 1. Escenario de aplicación para tarea NAP.

\section{Análisis de resultados}

Con las respuestas de los participantes se realizó el cálculo en proporción de las Normas de Asociación de palabras para cada grupo (Alzheimer, Demencia Mixta y sus Controles) con las medidas mencionadas anteriormente: fuerza de asociación (FA), el total de respuestas diferentes proporcionadas (NA), las respuestas idiosincrásicas o únicas (RI), respuestas en blanco (RB).

Se realizó un análisis $t$ de Student para grupos independientes para comparar los resultados de fuerza de asociación del primer asociado, el número de asociados diferentes, el porcentaje de respuestas idiosincrásicas y el porcentaje de respuestas en blanco por cada grupo y sus respectivos controles, los cuales fueron pareados por edad y escolaridad.

\section{Resultados}

\section{Enfermedad de Alzheimer vs. Controles}

Se observaron diferencias significativas entre los dos grupos al comparar el porcentaje de respuestas en blanco $(\mathrm{t}(238)=$ $6.84, p<0.05)$. El grupo con EA tuvo una mayor cantidad de 
respuestas en blanco (8.75 \pm 13.99$)$ que el grupo control, los cuales no presentaron respuestas en blanco (ver Figura 2). Por otro lado, en número de asociados diferentes se observaron diferencias significativas entre grupos $(\mathrm{t}(238)=2.71, \mathrm{p}<0.05)$, donde EA mostró menor cantidad de asociados (3.33 \pm 0.70$)$ que sus controles (3.57 \pm 3.56$)$. Finalmente, se encontraron diferencias significativas $(t(238)=16.33, p<0.05)$ en el tiempo de respuesta de los participantes, siendo aquellos con EA los que tenían mayores tiempos de respuesta $(14.66 \pm 6.67)$ que sus controles (4.56 \pm 1.20$)$.

En el caso de la fuerza de asociación del primer asociado, se observa una ligera diferencia entre ambos grupos ( $t$ (238) $=-1.38, p=0.17$ ), en la que el grupo con EA presentó menor fuerza de asociación del primer asociado (15.21 \pm 24.10$)$ que el grupo control $(19.79 \pm 27.29)$. Asimismo, se observan ligeras diferencias entre el porcentaje de respuestas idiosincrásicas ( $t$ $(238)=1.38, p=0.17$ ) que dieron ambos grupos de participantes, siendo aquellos con EA los que resultaron con mayor cantidad de respuestas de este tipo $(84.80 \pm 24.10)$ que sus controles (80.20 \pm 27.29$)$ (ver Figura 2).

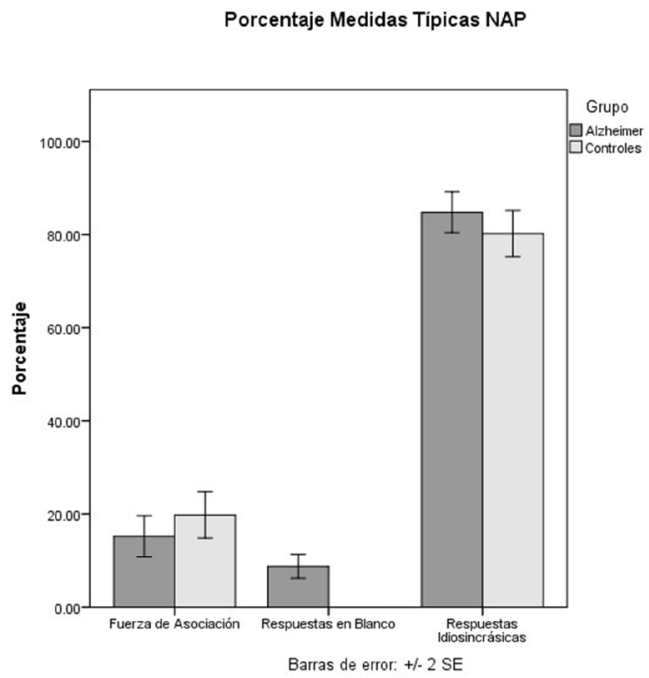

Figura 2. Porcentaje de Medidas Típicas de NAP 


\section{Demencia mixta vs. Controles}

Se observaron ligeras diferencias entre los dos grupos al comparar el porcentaje de respuestas en blanco de ambos grupos $(t(238)=0.59, p=0.556)$, en la que el grupo con MxD tuvo una mayor cantidad de respuestas en blanco $(1.94 \pm 7.84)$ que el grupo control $(1.39 \pm 6.68)$. Asimismo, en cuanto a porcentaje de respuestas idiosincrásicas, se observaron ligeras diferencias entre ambos grupos $(t(238)=1.58, p=0.11)$, siendo el grupo con $M \times D$ el que presenta mayor cantidad de respuestas de este tipo (86.94 $\pm 28.10)$ que sus controles (80.83 \pm 31.66$)$. Finalmente, en el tiempo de respuesta de los participantes fue similar $(t(238)=1.126, p$ $=2.61$ ), siendo aquellos con MxD los que tenían mayores tiempos de respuesta $(4.70 \pm 2.26)$ que sus controles $(4.82 \pm 2.34)$.

\section{Discusión}

Las Normas de Asociación de Palabras (NAP) son un corpus que describe la relación que existe entre dos palabras obtenidas a través de la técnica de asociación libre. Esta técnica permite obtener una lista de palabras evocadas por el sujeto y su tiempo de respuesta, además de la fuerza de asociación (FA) entre dos palabras, el total de respuestas diferentes proporcionadas (NA), las respuestas idiosincrásicas o únicas (RI) y las respuestas en blanco (RB). En este trabajo se utilizó esta técnica para obtener asociados de 120 palabras con ayuda de un sistema computarizado y, posteriormente, sus características en personas con EA, MxD y sus controles.

Las diferencias en el porcentaje de RB y el tiempo de reacción en pacientes con EA comparados contra los controles sugieren la existencia de dificultades para el acceso a las asociaciones léxicas en el grupo con enfermedad de Alzheimer, comparado con el grupo con demencia mixta y controles, lo cual se podría relacionar con la progresión de problemas en el lenguaje (Jaramillo, 2010). Estudios anteriores distinguen que las dificultades para acceder al conocimiento de la memoria semántica se manifiestan a través de: 1) dificultades para encontrar las palabras a las que se quieren referir; 2) aumento de los tiempos 
de respuesta, y 3) el uso de paráfrasis como una estrategia para compensar el déficit (Rabadán, De Juan y Rozas, 1998). Esto concuerda con nuestros resultados dado que, al existir dificultades para evocar dentro de la reserva léxica, aumentaron los tiempos de respuesta, así como mayor cantidad de respuestas en blanco en el grupo con EA.

El empleo de las Normas de Asociación de Palabras permite una aproximación pertinente al posible deterioro de la red léxica adulta en pacientes con neurodegeneración dado que nuestros datos, en lo concerniente a la comparación entre EA y envejecimiento típico, arrojan diferencias significativas en la mayoría de las medidas empleadas.

Una de las limitantes del presente trabajo es el número reducido de participantes; no obstante, en la actualidad nos encontramos en el proceso de ampliación de la muestra y control de variables, como el sexo, con el objetivo de continuar la exploración de las relaciones léxicas producidas en el contexto del envejecimiento típico y patológico.

La identificación temprana de cambios en las asociaciones léxicas podría servir como posibles indicadores de las alteraciones a nivel lingüístico que empiezan a desarrollarse desde etapas primarias ligadas al deterioro anatómico y fisiológico existente en los diversos tipos de demencia, los cuales, a su vez, podrían dar indicios sobre las características de los problemas en la memoria semántica y la evolución progresiva del cuadro demencial. Asimismo, trabajos futuros debieran categorizar el tipo de respuesta proporcionada con el objetivo de analizar los datos desde un enfoque cualitativo.

\section{Referencias}

Arango-Lasprilla, J., C., Fernández, G., S., Ardila, A. (2013). Las demencias: aspectos clínicos, neuropsicológicos y tratamiento. México: Manual Moderno.

Ardila, A. y Rosselli, M. (2007). Neuropsicología clínica. Editorial El Manual Moderno.

Ballesteros, J., S. (1999). Psicología de la memoria. Universidad Nacional de Educación a Distancia. 
Barrón-Martínez, J. B. y Arias-Trejo, N. (2014). Word Association Norms in Mexican Spanish. Spanish Journal of Psychology, 17(e90), 1-13.

Callejas, A., Correa, Á., Lupiáñez, J. y Tudela, P. (2003). Normas Asociativas Intracategoriales para 612 palabras de seis categorías semánticas en español. Psicológica, 24, 185-214. Cervantes, C. M., Alvarado, A. M., Navarro, S. A., Ávila, P. A., Gutiérrez, L. G., Arellano, S. J. y Funes, J. Á. (2017). Factores asociados a la demencia mixta en comparación con demencia tipo Alzheimer en adultos mayores mexicanos. Neurología, 32(5), 309-315.

Clark, H. H. (1970). Word associations and linguistic theory. In J. Lyons (Ed.), New Horizons in Linguistics, (pp. 271-286). Baltimore: Penguin.

Mendoza-Núñez, V. (2013). Envejecimiento y vejez. En V. M. Mendoza-Núñez, M. L. Martínez-Maldonado y L. A. Vargas Guadarrama (Eds.). Envejecimiento activo y saludable, (pp 51-55). México: UNAM, FES Zaragoza.

Peña-Casanova, J. (2007). Neurología de la conducta y neuropsicología. Ed. Médica Panamericana.

Pradilla, G. (2004). Enfermedad de Alzheimer. Salud UIS, 36, 146-153. http://doi.org/10.1016/S0304-5412(11)70068-3

Rabadán, O. J., de Juan, M. R. E. y Rozas, A. P. (1998). Problemas de acceso léxico en la vejez. Bases para la intervención. Anales de Psicología, 14(2), 169.

Tulving, E. (1972). Episodic and semantic memory. En E. Tulving y W. Donaldson. Organization of Memory (pp. 382-402). New York: Academic Press, Inc.

Villa, M., Navarro, M., y Villaseñor, T. (2017). Neuropsicología clínica hospitalaria. México: Manual Moderno. 